\title{
Pulmonary arterial hypertension in patients treated with interferon
}

\author{
Laurent Savale ${ }^{1,2,3}$, Caroline Sattler ${ }^{1,2,3}$, Sven Günther ${ }^{1,2,3}$, David Montani ${ }^{1,2,3}$, \\ Marie-Camille Chaumais ${ }^{3,4,5}$, Swanny Perrin ${ }^{3,4}$, Xavier Jaïs ${ }^{1,2,3}$, Andrei Seferian ${ }^{1,2,3}$, \\ Roland Jovan ${ }^{1,2,3}$, Sophie Bulifon ${ }^{1,2,3}$, Florence Parent ${ }^{1,2,3}$, Gérald Simonneau ${ }^{1,2,3}$, \\ Marc Humbert ${ }^{1,2,3}$ and Olivier Sitbon $n^{1,2,3}$
}

\begin{abstract}
Affiliations:
${ }^{1}$ Université Paris-Sud, Faculté de Médecine, Le Kremlin Bicêtre, France.

${ }^{2} \mathrm{AP}-\mathrm{HP}$, Centre de Référence de l'Hypertension Pulmonaire Sévère, Département Hospitalo-Universitaire (DHU) Thorax Innovation (TORINO), Service de Pneumologie, Hôpital de Bicêtre, Le Kremlin Bicêtre, France.

${ }^{3}$ UMR_S 999, INSERM, Laboratoire d'Excellence (LabEx) en Recherche sur le Médicament et l'Innovation Thérapeutique (LERMIT), Centre Chirurgical Marie Lannelongue, Le Plessis Robinson, France.

${ }^{4}$ Univ. Paris-sud, Faculté de Pharmacie, Châtenay Malabry, France.

${ }^{5} \mathrm{AP}-\mathrm{HP}$, Service de Pharmacie, Hôpital Antoine Béclère, Clamart, France.
\end{abstract}

Correspondence: Laurent Savale, Service de Pneumologie, Hôpital Universitaire de Bicêtre, 78, Rue du Général Leclerc, 94275 Le Kremlin-Bicêtre, France. E-mail: laurent.savaleabct.aphp.fr

ABSTRACT Isolated cases of pulmonary arterial hypertension (PAH) in patients treated with interferon (IFN) $\alpha$ or $\beta$ have been reported in the literature.

The aim of this study was to describe all consecutive cases of PAH patients with a history of IFN exposure identified in the French reference centre for severe pulmonary hypertension between 1998 and 2012.

A total of 53 patients with PAH and a history of IFN therapy were identified. 48 patients had been treated with IFN $\alpha$ for chronic hepatitis C. Most of them had portal hypertension (85\%) and 56\% had HIV coinfection. Five additional patients had been treated with IFN $\beta$ for multiple sclerosis. The diagnosis of PAH was made within 3 years after IFN therapy in $66 \%$ of patients. Repeated haemodynamic assessment was available in 13 out of 16 patients exposed to IFN after the diagnosis of PAH. Increased pulmonary vascular resistance $>20 \%$ was observed in 11 out of 13 cases (median $43 \%$ increase; IQR 32-67\%). In five of these patients, IFN withdrawal resulted in spontaneous haemodynamic improvement.

This retrospective analysis suggests that IFN therapy may trigger PAH. However, most of these patients had other risk factors for PAH. A prospective case-control study is necessary to definitively establish a link between IFN exposure and PAH.

0 @ERSpublications Clinical data suggest that interferon therapy may be a trigger for pulmonary arterial hypertension http://ow.ly/BweqG

\footnotetext{
This article has been revised according to the correction published in the December 2015 issue of the European Respiratory Journal.

Received: March 262014 | Accepted after revision: Aug 042014 | First published online: Oct 162014

Conflict of interest: Disclosures can be found alongside the online version of this article at erj.ersjournals.com 


\section{Introduction}

Pulmonary arterial hypertension (PAH) is a severe and progressive disease, characterised by elevated pulmonary artery pressure (PAP) and pulmonary vascular resistance (PVR) leading to right heart failure and ultimately death $[1,2]$. PAH may be idiopathic, heritable or associated with several conditions and diseases including connective tissue diseases, HIV infection, portal hypertension, congenital heart diseases and exposure to drugs and/or toxins $[3,4]$. Among drugs known to be associated with the development of $\mathrm{PAH}$, only anorectic drugs such as aminorex and fenfluramine derivatives, and toxic rapeseed oil are considered to be "definite" risk factors for PAH $[2,5]$. More recently, published data from the French registry of pulmonary hypertension has suggested that dasatinib, a tyrosine kinase inhibitor (TKI), may also induce PAH [6].

The role of interferon (IFN) is being increasingly considered a risk factor for the development of PAH. This is supported by the publication of several isolated case reports [7-14] and by experimental studies [15-18]. IFNs have powerful actions within the immune system where they play key anti-viral, anti-bacterial and anti-tumoural roles. They are classified as helical cytokines and categorised as type I or type II according to their physical and functional characteristics. Type I IFNs include $\alpha$ (released by leukocytes), $\beta$ (released by fibroblasts) and $\tau$ and $\varepsilon$ subtypes that likely arise from a common ancestral gene. According to their effects, IFNs have been used to treat a wide spectrum of diseases. IFN- $\alpha$ has been used extensively in the treatment of hepatitis viruses, but also in haematologic, nephrologic and dermatologic malignancies. The association of IFN- $\alpha$ and ribavirin has been considered to be the current standard of care for chronic hepatitis $\mathrm{C}$ virus (HCV) infection in the last decades. Recombinant IFN- $\beta$ is approved for the treatment of relapsingremitting multiple sclerosis [19].

The major drawback of IFN therapy is the occurrence of many various side effects, leading to poor patient compliance, resulting in reduced effectiveness. The leading adverse event affecting the respiratory system is cough (26\%) [20], while other side effects are rare and usually severe: interstitial lung disease (0.3\%) [21], pleural effusion, asthma exacerbations, granulomatosis, organised pneumonia and bilateral pulmonary infiltrates [14].

In the literature, 12 cases of pulmonary hypertension potentially associated with IFN exposure have been described [7-14]. Moreover, recent experimental studies have suggested that IFN could promote endothelial dysfunction increasing the release of endothelin-1 by human pulmonary vascular cells [16]. On the basis of these findings, our local PAH registry was consulted for patients who had been exposed to IFN, in order to assess a potential link between IFN and PAH.

The present report summarises the clinical characteristics and outcome of consecutive patients with PAH associated with a history of IFN exposure in the French reference centre for severe pulmonary hypertension $(\mathrm{PH})$ at Paris-Sud University.

\section{Methods}

Patients

The French Registry of patients with PAH served as the main database for selecting PAH patients who had been exposed to IFN. We restricted the search to patients followed-up at the Reference Centre (Paris-Sud University). This retrospective study complies with the Declaration of Helsinki. Although French law does not require ethics committee approval or informed consent for retrospective data collection, the data were anonymised and complied with the requirements of the Commission Nationale Informatique et Liberté, the organisation responsible for privacy, information technology and civil rights in France. The committee approved the methods used to collect and analyse data on May 24, 2003 (approval number 842063).

All patients with PAH (i.e. group 1 of the clinical classification of $\mathrm{PH}$ ) [4] with a history of IFN exposure were included in the analysis. $\mathrm{PH}$ was defined as mean pulmonary arterial pressure (mPAP) $\geqslant 25 \mathrm{mmHg}$ and pulmonary artery wedge pressure $(\mathrm{PAWP}) \leqslant 15 \mathrm{mmHg}$ measured with right heart catheterisation. All patients whose diagnosis of $\mathrm{PH}$ was confirmed by right heart catheterisation underwent extensive investigations in order to identify additional causes or risk factors for vascular disorders (congenital heart disease, porto-pulmonary hypertension, HIV infection, connective tissue disease, chronic thrombo-embolic $\mathrm{PH}$, chronic obstructive or restrictive lung disease, systolic or diastolic left heart disease, haemoglobinopathies, etc.). Age at diagnosis and clinical status as assessed by modified New York Heart Association (NYHA) functional class were recorded at diagnosis. A non-encouraged 6-min walk test, as recommended by the American Thoracic Society, was performed.

Patients with chronic myelogenous leukaemia, exposed to IFN and dasatinib were excluded because dasatinib has recently been incriminated for inducing PAH [6]. 


\section{IFN exposure}

The specifics of IFN exposure were gathered by detailed clinical history either at time of initial work-up or $a$ posteriori depending on each case. The type of IFN ( $\alpha$ or $\beta$ ), the date of initial exposure and duration of total exposure were established after interviews with patients and their hepatologists.

\section{Statistical analysis}

Data were stored on a PC-based data spreadsheet. Analysis was performed using the statistical package StatEL (Ad Science, Paris, France) on Microsoft Excel 2007. All data are expressed as mean \pm SD or median and interquartile range (IQR) as appropriate. In patients with already-diagnosed $\mathrm{PAH}$ who were exposed to IFN, a comparison of haemodynamic variables obtained at last evaluation before IFN exposure, on IFN and after IFN withdrawal was made using one-way ANOVA for paired groups for normally distributed variables and a non-parametric Friedman test for the non-normally distributed variables. Post hoc comparisons were made using the Newman and Keuls test or the non-parametric Wilcoxon test, as appropriate. A p-value $<0.05$ was considered as statistically significant.

\section{Results}

Study population

From 1998 to 2012, 53 PAH patients with a history of IFN exposure were identified in the French reference centre for severe $\mathrm{PH}$. In 48 of them, $\mathrm{PAH}$ was diagnosed after exposure to IFN. In the remaining five patients, IFN therapy was started while PAH was already known.

\section{Patients treated with IFN- $\alpha$}

Among the study population, 47 had been treated with IFN- $\alpha$ for chronic HCV infection and one for chronic myelogenous leukaemia. Median (IQR) duration of treatment with IFN- $\alpha$ was 7.7 months (5.3-11.0 months). IFN- $\alpha 2$ a was used in $80 \%$ of cases and IFN- $\alpha 2 \mathrm{~b}$ in $20 \%$ of cases. Among these 48 patients, eight $(17 \%)$ were still on IFN- $\alpha$ at the time of PAH diagnosis. PAH was diagnosed within 3 years following IFN- $\alpha$ exposure in $35(73 \%)$ out of 48 patients (fig. 1).

Clinical and haemodynamic characteristics of the 48 IFN- $\alpha$-treated patients assessed at the time of PAH diagnosis are shown in table 1. All patients with cirrhosis had evidence of portal hypertension as documented by the presence of at least one of the following: ascites, oesophageal varices or elevated hepatic venous pressure gradient ( $>5 \mathrm{mmHg}) .27(56 \%)$ patients had HIV co-infection. The haemodynamics of HCV patients exposed to IFN, with or without HIV co-infection, were similar to that of portopulmonary hypertension patients from the French registry (data not shown).

\section{Patients treated with IFN- $\beta$}

Five patients were treated with IFN- $\beta$ for multiple sclerosis at the time of PAH diagnosis. In those patients, the time between initiation of IFN- $\beta$ and diagnosis of PAH ranged from 59 to 117 months (fig. 1, table 2). Individual characteristics of these five patients are detailed in table 2. One of these patients had an atrial septal defect that is a definite risk factor for PAH. Most of them developed severe PAH, leading to death in two cases.

\section{The effect of IFN on PAH progression}

16 patients were treated with IFN- $\alpha$ while the diagnosis of PAH was known. IFN was administered for this first time in five patients while 11 had already been treated in the past. At the time of IFN initiation, eight out of 16 patients were on PAH-specific therapies (endothelin-receptor antagonist and/or phosphodiesterase type 5 inhibitor). Clinical and haemodynamic characteristics of these patients are shown in table 3 . In those patients, the median duration of IFN exposure was 10 months (IQR 6.4-12.9). 13 were reassessed while undergoing IFN therapy (median 7.4 months; IQR 6.4-13.8) and following IFN discontinuation (median 7.7 months; IQR 6.0-8.9). The course of NYHA functional class, 6-min walk distance (6MWD) and pulmonary vascular resistance (PVR) is shown on figure 2. The increase in PVR was more than $20 \%$ in 11 out of 13 patients (from $5 \pm 2.1$ to $8.1 \pm 3.2$; $\mathrm{p}<0.05$ ). This haemodynamic deterioration was associated with worsening of functional and exercise capacity (fig. 2). Four patients needed additional PAH-specific therapy following clinical and haemodynamic worsening while on IFN. In five other patients, IFN withdrawal resulted in clinical and haemodynamic improvements without any additional therapy. In contrast, no improvement of PAH was observed after IFN withdrawal in the two remaining patients.

\section{Discussion}

Since 1998, the French reference centre for severe PH has identified 53 cases of symptomatic precapillary $\mathrm{PAH}$ in patients treated with IFN. The vast majority of patients had been treated with IFN- $\alpha$ for chronic 


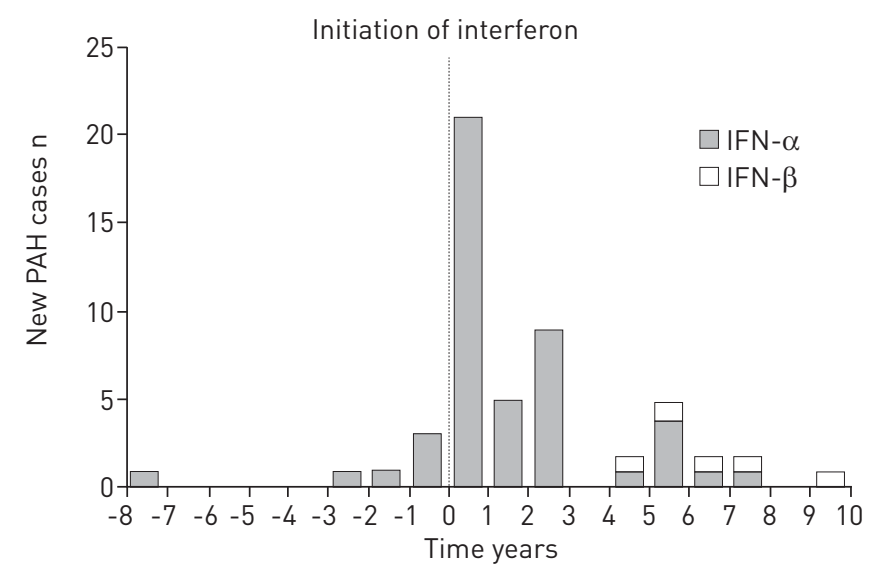

FIGURE 1 Delay between interferon (IFN) commencement and pulmonary arterial hypertension (PAH) diagnosis. Twothirds of PAH cases were diagnosed within 3 years after initiation of IFN therapy. In five patients receiving IFN- $\beta$ therapy, PAH was diagnosed 5-10 years after commencement of exposure.

HCV infection. All of them had another risk factor for PAH (portal hypertension and/or HIV infection). Five patients received IFN- $\beta$ for multiple sclerosis among which only one had another risk factor for PAH.

Despite the presence of other risk factors associated with PAH in most patients, several characteristics suggest a potential link between exposure to IFN and the occurrence of PAH. First of all, we observed that two-thirds of the patients were diagnosed with PAH within 3 years after IFN initiation. The time delay was longer in patients treated with IFN- $\beta$. We also observed worsening of symptoms, exercise capacity and haemodynamic parameters in the majority of patients with PAH who had been exposed to IFN. Finally, the description of PAH in patients without any other risk factors for the disease emphasises the potential role of IFN in the development of PAH.

12 other cases of PAH potentially associated with IFN therapy have been reported in the literature [7-13]; only five of which were diagnosed using right heart catheterisation. The first case, published in 1993, described reversible acute right heart failure in a patient with renal cell carcinoma after IFN therapy,

\section{TABLE 1 Baseline characteristics of patients treated with IFN- $\alpha$}

$\begin{array}{lc}\text { Subjects } \mathbf{n} & 48 \\ \text { Age years } & 46(41-50) \\ \text { Sex } & \\ \text { Female } & 15(31) \\ \text { Male } & 33(69) \\ \text { IFN exposure duration months } & 7.7(5.3-11.0) \\ \text { HCV infection } & 47(98) \\ \text { HBV infection } & 5(10) \\ \text { HIV co-infection } & 27(56) \\ \text { Cirrhosis } & 41(85) \\ \text { NYHA-FC } & 15(31) \\ \text { I-II } & 33(69) \\ \text { III-IV } & 425(347-454) \\ \text { 6MWD m } & \\ \text { Haemodynamics } & 7 \pm 5 \\ \text { RAP mmHg } & 46 \pm 10 \\ \text { mPAP mmHg } & 8 \pm 3 \\ \text { PAWP mmHg } & 5.5 \pm 1.5 \\ \text { Cardiac output L·min } & \\ \text { PVR Wood Units } & 7.7 \pm 3.6\end{array}$

Data are presented as median (interquartile range), $\mathrm{n}(\%)$ or mean $\pm \mathrm{SD}$. IFN: interferon; HCV: hepatitis $\mathrm{C}$ virus; HBV: hepatitis B virus; HIV: human immunodeficiency virus; NYHA-FC: New York Heart Association Functional Class; 6MWD: 6-min walk distance; RAP: right atrial pressure; mPAP: mean pulmonary artery pressure; PAWP: pulmonary artery wedged pressure; PVR: pulmonary vascular resistance. 
TABLE 2 Individual characteristics of patients treated with IFN- $\beta$

\begin{tabular}{|c|c|c|c|c|c|c|c|}
\hline Patient & Sex & Age years & $\begin{array}{l}\text { Delay between } \\
\text { initiation of IFN- } \beta \\
\text { therapy and } \\
\text { diagnosis of PAH }\end{array}$ & mPAP $\mathrm{mmHg}$ & $\begin{array}{l}\text { Cardiac output } \\
\qquad \mathrm{L} \cdot \mathrm{min}^{-1}\end{array}$ & PVR Wood Units & Outcome \\
\hline $1^{\#}$ & $\mathrm{~F}$ & 41 & 65 months & 52 & 9.3 & 3.1 & Alive \\
\hline 2 & $\mathrm{~F}$ & 45 & 80 months & 52 & 3.8 & 11.7 & Alive \\
\hline 3 & $\mathrm{~F}$ & 48 & 95 months & 69 & 4.4 & 11.3 & Alive \\
\hline
\end{tabular}

\#: Patient 1 has an atrial septal defect (ASD) as another risk factor for pulmonary arterial hypertension (PAH). ASD was closed and interferon (IFN) discontinued after diagnosis of PAH leading to stabilisation of the disease. mPAP: mean pulmonary artery pressure; PVR: pulmonary vascular resistance.

suggesting for the first time a possible causative mechanism [13]. Overall, 10 cases were attributed to IFN- $\alpha$ and two to IFN- $\beta$. Only two cases were considered to be reversible after IFN withdrawal. In contrast, the 10 other cases needed the use of PAH-specific therapy or led to death. Interestingly, all cases in the literature were diagnosed within 3 years after initiation of IFN therapy, which is in line with our observations in patients exposed to IFN- $\alpha$. In other clinical studies, there is growing evidence that PAH could occur or worsen with IFN therapy. A decrease by at least $15 \%$ of the diffusing capacity of the lung for CO in as many as $48 \%$ of patients treated with IFN- $\alpha$ for chronic HCV infection has been observed. This suggests an underlying pulmonary vascular pathology induced by this treatment [22]. Furthermore, serum level of endothelin-1, a key mediator in the pathogenesis of $\mathrm{PAH}$, is elevated in patients receiving IFN $\alpha$ therapy for hepatitis $\mathrm{C}$ infection [23]. The strength of our report is the availability of both a large prospective PAH database that allows surveillance of trends for PAH risk factors and invasive pulmonary haemodynamic data at baseline and following IFN withdrawal.

The temporal patterns of drug-induced PAH are not always highly suggestive of a causal role, without ruling out the responsibility of the drug in the occurrence of the disease. Experience with anorectic agents has shown that the time to onset of pulmonary vascular damage after initiation of treatment can sometimes be very long (median $>5$ years) and can occur away from the cessation of exposure [24, 25]. This property, combined with a small proportion of patients affected by the side effect, partly explained late recognition of the role of some drugs in the emergence of new cases of PAH. More recently, the reported cases of dasatinib-induced PAH has shown the possibility of a shorter time between drug exposure and PAH occurrence (34 months) [6]. In our study, we observed a chronological association that suggests accountability of IFN in the onset of PAH. The involvement of IFN has been formally ruled out in five patients because PAH was known before the start of exposure. In these patients we only analysed the effect of IFN on PAH progression. In the majority of patients treated with IFN- $\alpha$, PAH was diagnosed within 3 years following drug exposure. It is interesting to note that the time between exposure to IFN- $\beta$ and the diagnosis of PAH was much longer (5-10 years). These cases are however of importance as no confounding risk factor for PAH has been found in four of them. Hypotheses can be drawn to explain this longer time period between IFN- $\beta$ exposure and occurrence of PAH. The biological activities of IFN- $\alpha$ and IFN- $\beta$ are distinct. We can assume that there are differences between these molecules on their potentiality to induce endothelial dysfunction and pulmonary vascular remodelling. Further experimental studies are needed to investigate this possible association.

We can also assume that IFN- $\alpha$ could play the role of a trigger by precipitating the development of pulmonary vascular disease in a shorter delay in predisposed patients. Several characteristics of druginduced PAH suggested in the past that a drug could play the role of a second hit. This was especially shown with anorectic agents. The presence of other risk factors associated with the disease has been reported in a relatively high proportion of patients exposed to fenfluramine and derivatives [24, 26]. Moreover, in this population of PAH associated with anorexigens, the proportion of patients carrying the BMPR2 mutation has been found to be the same as patients with idiopathic PAH [25]. In addition, the time to drug exposure was shorter in patients carrying the BMPR2 mutation when compared with those who did not [25]. Similarly, the high proportion of patients exposed to IFN who had another risk factor for PAH should not exclude the accountability of IFN in the development of PAH. 

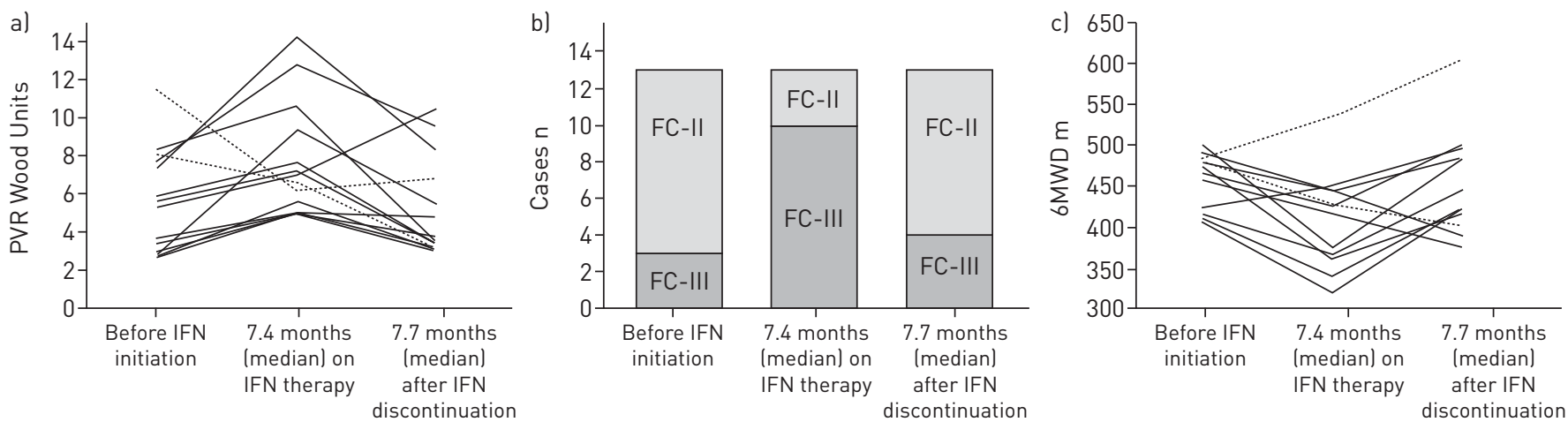

FIGURE 2 Effect of interferon (IFN)- $\alpha$ on clinical, functional and haemodynamic parameters in patients with pulmonary arterial hypertension. Evolution of clinical, functional and haemodynamic parameters at median 7.4 months (interquartile range (IQR) 6.4-13.8) after IFN initiation and 7.7 months (IQR, 6-8.9) after IFN withdrawal. The last evaluation before IFN initiation was performed 3.7 months (IQR, 1.2-9.2) before hepatitis C virus treatment. After IFN initiation, a worsening of b) New York Heart Association functional class (FC) was observed and c) 6-min walk distance (6MWD) significantly decreased. The invasive haemodynamic evaluation showed significant increase in pulmonary vascular resistance (PVR) $(\mathrm{p}<0.05)$. a) PVR decreased after IFN initiation in only two patients (dashed line). PAH-specific therapy was reinforced in four patients after IFN initiation. Functional, clinical and haemodynamic evaluation at distance of IFN withdrawal showed spontaneous resolution of worsening in five patients without need for additional PAH-specific therapy.

IFN is a common mediator that can be unregulated in many conditions associated with higher risk to develop PAH such as connective tissue diseases or viral infection. An "IFN signature" characterised by increase expression and activation of IFN-regulated genes has been described in circulating monocytes in patients with systemic scleroderma or systemic lupus erythematosus [27]. Interestingly, a placebocontrolled trial evaluating the effect of IFN on systemic sclerosis, suggested that IFN- $\alpha$ was, in fact, of no value in the treatment of scleroderma and may be deleterious. In the group of patients treated with active therapy, a greater deterioration of lung function and diffusing capacity for carbon monoxide was observed [28]. Later, it was suggested that IFN may be involved in the development of fibrosis or PAH in patients with scleroderma [29]. Similarly, it has been suggested that HIV infection could promote PAH through indirect mechanisms and release of inflammatory mediators or growth factors. There is a chronic production of IFN $\alpha$ in patients with HIV infection [30,31] and an increased IFN expression correlates with HIV disease progression [32]. It is interesting to note that the proportion of patients with HIV co-infection in our study is higher than it is usually observed in patients with chronic HCV infection [33]. Because endogenous IFN is involved in the physiopathology of these conditions, exogenous effect of IFN is much harder to define. We could therefore speculate that these patients could be more prompt to develop PAH when exposed to exogenous IFN due to underlying predispositions.

TABLE 3 Clinical and haemodynamic characteristics of patients with pulmonary arterial hypertension already diagnosed at last evaluation before IFN exposure

$\begin{array}{lc}\text { Subjects } & 16 \\ \text { Age years } & 46(40-51) \\ \text { Sex } & \\ \text { Female } & 2(13) \\ \text { Male } & 14(87) \\ \text { NYHA-FC } & 13(81) \\ \text { I-II } & 3(19) \\ \text { III-IV } & 460(409-479) \\ \text { 6MWD m } & \\ \text { Haemodynamics } & 5 \pm 3 \\ \text { RAP mmHg } & 42 \pm 11 \\ \text { mPAP mmHg } & 7 \pm 3 \\ \text { PAWP mmHg } & 6.8 \pm 1.7 \\ \text { Cardiac output L·min }{ }^{-1} & 5.8 \pm 2.6 \\ \text { PVR Wood Units } & \end{array}$

Data are presented as $n$, median (interquartile range), $n(\%)$ or mean \pm SD. NYHA-FC: New York Heart Association Functional Class; 6MWD: 6-min walk distance; RAP: right atrial pressure; MPAP: mean pulmonary artery pressure; PAWP: pulmonary artery wedged pressure; PVR: pulmonary vascular resistance. 
Cases of PAH without any risk factors are unusual and mostly observed with IFN- $\beta$. However, these cases are very important because they reinforce the idea of a potential link between IFN exposure and the development of PAH.

The analysis of the effect of IFN on PAH progression provided interesting additional information. We obtained comprehensive and reliable data for 13 patients with available haemodynamic parameters. The haemodynamic deterioration observed in 11 out of 13 patients after exposure to IFN reinforces the hypothesis of accountability of IFN in PAH. More interestingly, this deterioration in haemodynamics was reversible after IFN discontinuation in most of these patients, without reinforcement of PAH-specific therapy in half of the cases.

Many experimental studies reinforced the hypothesis of a link between IFN exposure and development of PAH. As experimental investigations in sheep have shown that IFN- $\alpha$ was able to stimulate the thromboxane cascade resulting in transient $\mathrm{PH}$ [34], the authors suggested that IFN might accelerate a subclinical phenomenon caused by other factors such as the human herpes virus 8 , the hepatitis $\mathrm{C}$ virus itself, or an unknown genetic predisposition. Other studies reported a link between IFN and the release of endothelin (ET-1), a key mediator involved in the pathogenesis of PAH. It has been shown that IFN could induce ET-1 in TNF- $\alpha$-primed human pulmonary artery smooth muscle cells [18]. The discovery of a "sensitising" effect of TNF on the IFN responsiveness in human pulmonary artery smooth muscle cells begs the question of whether underlying inflammatory conditions could predispose to PAH development. This experimental discovery could explain why IFN seems to induce a more deleterious effect on the pulmonary circulation in inflammatory diseases, such as portal hypertension or HIV infection. More recently, it has been demonstrated that type I IFN receptor knockout (IFNAR1(-/-)) mice were protected from the effects of hypoxia on the right heart, vascular remodelling, and raised serum ET-1 levels. These data suggested that type I IFN could mediate PAH via an action of IFNAR1 [15]. Further studies are needed to establish the effect of IFN on other animal models of $\mathrm{PH}$, such as $\mathrm{PH}$ induced by monocrotaline or chronic hypoxia.

Over the past decade, the development of a national network and the creation of a web-based registry have facilitated the detection of drugs potentially associated with PAH. This network may help to detect potential risk factors associated with PAH by analysing pathophysiological, epidemiological and historical data. Thereafter, experimental and clinical studies may be undertaken to validate or reject the hypothesis. This approach requires close collaboration with pharmacovigilance centres and national drug regulatory agencies in order to compare available data, assess level of accountability and to define necessary actions to reduce the risk of PAH associated with the drug. Our clinical study provided additional information to what was known from the literature about a possible link between IFN exposure and occurrence of PAH. However, our study has some limitations mainly due to its retrospective and monocentric nature, such as the existence of confounding risk factors for PAH in patients exposed to IFN- $\alpha$. It might be useful to study the feasibility of a case-control study to establish a definite link between IFN exposure and PAH development and estimate the incidence of PAH development in patients exposed to IFN. However, this treatment may be supplemented with other therapeutic options for viral hepatitis in the coming years [35].

Results of this study may provide some recommendations for the management of IFN therapy in patients with HCV infection. Among patients without diagnosed PAH at the time of IFN initiation, the occurrence of unexplained dyspnoea and/or signs of right ventricular failure on IFN should lead to PAH detection. If $\mathrm{PAH}$ is confirmed, IFN should be discontinued and PAH-specific therapy must be considered. In patients with diagnosed PAH and an indication for IFN therapy for HCV infection, an extensive work-up including a right heart catheterisation and exercise tests has to be performed before initiation of any IFN therapy. In stable, non-severe PAH patients, IFN can be administrated in parallel with a close monitoring of the pulmonary vascular disease. If control of $\mathrm{PAH}$ is unsatisfactory, intensification of $\mathrm{PAH}$-specific therapy has to be considered before IFN initiation.

In conclusion, IFN could act as a "trigger" in patients with predisposed conditions to develop PAH. However, "pure" cases of PAH have been identified in patients without any risk factors for PAH and treated with IFN- $\beta$. According to the current clinical classification of PH, IFN is now considered a "possible" risk factor for PAH [4]. Our study highlights the need for clinical and experimental research to determine the association of IFN exposure and the development of PAH to have a better understanding of the underlying mechanisms in order to prevent side effects in the pulmonary vasculature.

\section{Acknowledgements}

We acknowledge the French pulmonary hypertension pharmacovigilance network, VIGIAPATH, supported by the Agence Nationale de Sécurité du Médicament et des Produits de Santé (ANSM). 


\section{References}

Rubin L. Primary pulmonary hypertension. N Engl J Med 1997; 336: 111-117.

Simonneau G, Galie N, Rubin LJ, et al. Clinical classification of pulmonary hypertension. J Am Coll Cardiol 2004; 43: $5 \mathrm{~S}-12 \mathrm{~S}$

3 Simonneau G, Robbins IM, Beghetti M, et al. Updated clinical classification of pulmonary hypertension. J Am Coll Cardiol 2009; 54: S43-S54.

4 Simonneau G, Gatzoulis MA, Adatia I, et al. Updated clinical classification of pulmonary hypertension. J Am Coll Cardiol 2013; 62: D34-D41.

Fishman AP. Clinical classification of pulmonary hypertension. Clin Chest Med 2001; 22: 385-391.

6 Montani D, Bergot E, Gunther S, et al. Pulmonary arterial hypertension in patients treated by dasatinib. Circulation 2012; 125: 2128-2137.

7 Caravita S, Secchi MB, Wu SC, et al. Sildenafil therapy for interferon-beta-1a-induced pulmonary arterial hypertension: a case report. Cardiology 2011; 120: 187-189.

8 Dhillon S, Kaker A, Dosanjh A, et al. Irreversible pulmonary hypertension associated with the use of interferon alpha for chronic hepatitis C. Dig Dis Sci 2010; 55: 1785-1790.

9 Ledinek AH, Jazbec SS, Drinovec I, et al. Pulmonary arterial hypertension associated with interferon beta treatment for multiple sclerosis: a case report. Mult Scler 2009; 15: 885-886.

10 Jochmann N, Kiecker F, Borges AC, et al. Long-term therapy of interferon-alpha induced pulmonary arterial hypertension with different PDE-5 inhibitors: a case report. Cardiovasc Ultrasound 2005; 3: 26.

11 Al-Zahrani H, Gupta V, Minden MD, et al. Vascular events associated with alpha interferon therapy. Leuk Lymphoma 2003; 44: 471-475.

12 Fruehauf S, Steiger S, Topaly J, et al. Pulmonary artery hypertension during interferon- $\alpha$ therapy for chronic myelogenous leukemia. Ann Hematol 2001; 80: 308-310.

13 Kramers C, de Mulder PH, Barth JD, et al. Acute right ventricular heart failure in a patient with renal cell carcinoma after interferon therapy. Neth J Med 1993; 42: 65-68.

14 Anderson P, Hoglund M, Rodjer S. Pulmonary side effects of interferon- $\alpha$ therapy in patients with hematological malignancies. Am J Hematol 2003; 73: 54-58.

15 George PM, Oliver E, Dorfmuller P, et al. Evidence for the involvement of type I interferon in pulmonary arterial hypertension. Circ Res 2014; 114: 677-688.

16 Woods M, Wood EG, Mitchell JA, et al. Signal transduction pathways involved in cytokine stimulation of endothelin-1 release from human vascular smooth muscle cells. J Cardiovasc Pharmacol 2000; 36: S407-S409.

17 Woods M, Wood EG, Bardswell SC, et al. Role for nuclear factor- $\mathrm{\kappa B}$ and signal transducer and activator of transcription 1/interferon regulatory factor-1 in cytokine-induced endothelin-1 release in human vascular smooth muscle cells. Mol Pharmacol 2003; 64: 923-931.

18 Badiger R, Mitchell JA, Gashaw H, et al. Effect of different interferonalpha2 preparations on IP10 and ET-1 release from human lung cells. PLoS One 2012; 7: e46779.

19 George PM, Badiger R, Alazawi W, et al. Pharmacology and therapeutic potential of interferons. Pharmacol Ther 2012; 135: 44-53.

20 Hezode C, Forestier N, Dusheiko G, et al. Telaprevir and peginterferon with or without ribavirin for chronic HCV infection. N Engl J Med 2009; 360: 1839-1850.

21 Ji FP, Li ZX, Deng H, et al. Diagnosis and management of interstitial pneumonitis associated with interferon therapy for chronic hepatitis C. World J Gastroenterol 2010; 16: 4394-4399.

22 Foster GR, Zeuzem S, Pianko S, et al. Decline in pulmonary function during chronic hepatitis C virus therapy with modified interferon alfa and ribavirin. J Viral Hepat 2013; 20: e115-e123.

23 George PM, Cunningham ME, Galloway-Phillipps N, et al. Endothelin-1 as a mediator and potential biomarker for interferon induced pulmonary toxicity. Pulm Circ 2012; 2: 501-504.

24 Savale L, Chaumais MC, Cottin V, et al. Pulmonary hypertension associated with benfluorex exposure. Eur Respir J 2012; 40: 1164-1172.

25 Souza R, Humbert M, Sztrymf B, et al. Pulmonary arterial hypertension associated with fenfluramine exposure: report of 109 cases. Eur Respir J 2008; 31: 343-348.

26 Rich S, Rubin L, Walker AM, et al. Anorexigens and pulmonary hypertension in the United States: results from the surveillance of North American pulmonary hypertension. Chest 2000; 117: 870-874.

27 Assassi S, Mayes MD, Arnett FC, et al. Systemic sclerosis and lupus: points in an interferon-mediated continuum. Arthritis Rheum 2010; 62: 589-598.

28 Black CM, Silman AJ, Herrick AI, et al. Interferon- $\alpha$ does not improve outcome at one year in patients with diffuse cutaneous scleroderma: results of a randomized, double-blind, placebo-controlled trial. Arthritis Rheum 1999; 42: 299-305.

29 Eloranta ML, Franck-Larsson K, Lovgren T, et al. Type I interferon system activation and association with disease manifestations in systemic sclerosis. Ann Rheum Dis 2010; 69: 1396-1402.

30 Zagury D, Lachgar $\mathrm{A}$, Chams $\mathrm{V}$, et al. Interferon alpha and Tat involvement in the immunosuppression of uninfected T cells and C-C chemokine decline in AIDS. Proc Natl Acad Sci USA 1998; 95: 3851-3856.

31 Lehmann C, Lafferty M, Garzino-Demo A, et al. Plasmacytoid dendritic cells accumulate and secrete interferon alpha in lymph nodes of HIV-1 patients. PLoS One 2010; 5: e11110.

32 Mandl JN, Barry AP, Vanderford TH, et al. Divergent TLR7 and TLR9 signaling and type I interferon production distinguish pathogenic and nonpathogenic AIDS virus infections. Nat Med 2008; 14: 1077-1087.

33 Operskalski EA, Kovacs A. HIV/HCV co-infection: pathogenesis, clinical complications, treatment, and new therapeutic technologies. Curr HIV/AIDS Rep 2011; 8: 12-22.

34 Hanaoka M, Kubo K, Hayano T, et al. Interferon-alpha elevates pulmonary blood pressure in sheep-the role of thromboxane cascade. Eur J Pharmacol 1999; 370: 145-151.

35 Kowdley KV, Lawitz E, Poordad F, et al. Phase $2 \mathrm{~b}$ trial of interferon-free therapy for hepatitis C virus genotype 1. N Engl J Med 2014; 370: 222-232. 